\title{
The Laboratory Should Actively be Involved in the Therapeutic Drug Monitoring (TDM) Process
}

\author{
Ozgur Aydin"1*, Hamit Yasar Ellidag², Esin Eren³, Necat Yilmaz ${ }^{2}$ \\ ${ }^{1}$ Biochemistry Laboratory, Batman Maternity and Children's Hospital, Batman, TURKEY. \\ ${ }^{2}$ Central Laboratories of Antalya Education and Research Hospital of Ministry of Health, Antalya, TURKEY. \\ ${ }^{3}$ Biochemistry Laboratory, Antalya Public Health Center of Ministry of Health, Antalya, TURKEY.
}

\begin{abstract}
Background: After forty years, the literature on therapeutic drug monitoring (TDM) is still contradictory. Researchers need to share their experiences to build up a concencus on basics of TDM. Material and Methods: The electronic database between $1 / 3 / 2013$ and $31 / 12 / 2013$ was retrospectively searched for information about TDM for carbamazepine, valproic acid, phenytoin and digoxin serum level measurements. Results: 184 carbamazepine, 283 valproic acid, 64 phenytoin and 135 digoxin tests were performed. Neurology Department requested $59 \%$ of total anti-epiteptics. $48 \%$ of digoxin requests were from Emergency and Intensive Care Units. 101 (55\%) of carbamazepine results and $43(68 \%)$ of phenytoin results were in the sub-therapeutic range; $158(56 \%)$ of valproic acid results were in the therapeutic and $55(41 \%)$ of digoxin results were in above therapeutic range. Conclusion: The study results suggested that digoxin tests were mostly requested to confirm drug toxicity while neuorologic drug requests mostly aimed to manage drug dosage. Percentage of test requests were in line with some of the previous studies and contrary to some others. A request form including the information about the drug dosage and dosing interval, the patient's response, indication for TDM is strongly suggested by the authors of this study. The laboratory should be able to read this basic information in order to be actively involved in test process.
\end{abstract}

Key words: Therapeutic, Drug monitoring, Carbamazepine, Valproic acid, Phenytoin, Digoxin.

\section{INTRODUCTION}

Therapeutic drug monitoring (TDM) was started as an attempt to protect patients against drug toxicity. Digoxin assays were the first to emerge in the market and used by the laboratories. ${ }^{1}$ After five decades, TDM has evolved as a multidiciplinary field of medicine where laboratory and clinical specialists join on the common purpose of personalizing the dosage of therapeutic agents. ${ }^{2,3}$ Anti-epileptic drugs (AEDs) are perfectly suitable for TDM due to their narrow therapeutic range, and marked inter-individual pharmacokinetic variability. Till date, there are no clear definitions that can guide the clinician to decide on a specific AED with a specific dosage on an individual patient. When initiating a medical therapy, the clinician is almost blind to predict if the patient will respond well to the drug of choice or experience unwanted drug effects. At this point TDM aims to contribute to the decision making.

The medical staff is responsible against the patient to manage the therapy with the most appropriate dosage of drug. Digoxin is used by the elderly, a population with physiologic multiorgan malfunctions that label every patient with a potential of unexpected response to medication. In case of digoxin, the major concern has been drug toxicity. When dealing with AED, TDM requires more than simply measuring patients blood drug concentrations and compare them to a target range, but to keep the patient safe from convulsions as long as possible or ideally eliminate the disease with the minimal dosage of the drug used. It is called "individualization" of drug dosage. ${ }^{4}$ In TDM practice,
Submitted date : 12/07/2015 Accepted date : 09/02/2016

DOI: 10.5530/ijopp.9.1.3

Address for correspondence: Dr. Ozgur Aydin, Biochemistry Laboratory Batman Maternity and Children's Hospital, Ziya Gökalp Mah. SSK Caddesi, Batman-Turkey. E-mail: belozgur@hotmail.com 
the clinician tries to maintain serum drug concentrations within a pre-defined therapeutic range, which defines the highest probability that the patient will benefit from the drug and be safe from side effects. However, it should not be forgotten that these ranges are statistical findings and there will always be patients who recover with sub-therapeutic dosages and who will suffer side effects or even toxicity within therapeutic levels.

There is a debate on the efficiency of TDM in the literature. ${ }^{5,6}$ The utility of TDM has not been clearly established. TDM is costly and pressure continues within the healthcare system of many countries to provide services at the lowest possible cost. Rational utilisation of TDM is an exact partner of drug dosage individualization. When used appropriately, it may improve patient management by maximising disease control and minimising the risk of adverse drug reactions; finally have a cost-saving effect. ${ }^{3}$ To optimize the use of TDM in laboratory, it is essential for the TDM performers to share experiences. The present study shares our experience with TDM for phenytoin, valproic acid, carbamazepine and digoxin in a period of 10 months.

\section{MATERIAL AND METHODS}

The study was conducted in a community hospital which serves as a teaching hospital and also as a tertiary care referral centre with 950 bed capacity. The central laboratory facilitates the needs of the entire hospital including smaller medical units. TDM was initially run by the Clinical Pharmacology Department between 2010 and 2011. After January 2011 TDM unit was moved to the Central Laboratory under the control of Clinical Biochemistry. TDM test panel in our hospital currently includes phenytoin, carbamazepine, valproic acid and digoxin.

This is a retrospective analysis of the existing electronic database. TDM tests were identified through a code search between $1 / 3 / 2013$ and $31 / 12 / 2013$. Database search was conducted to collect the information such as name, age and gender of the patients, the unit requesting the test.

After the test was requested by the clinician, the patients were sent to the sample stations where the blood was collected by direct puncture to a vein. Blood was drawn into a $4 \mathrm{~mL}$ serum tube with a gel seperator. Serum was separated by centrifuging the blood at $3000 \mathrm{rpm}$ for 10 minutes. The Beckman carbamazepine, valproic acid and phenytoin assays are based on the bacterial enzyme $\beta$-galactosidase, which has been genetically engineered into two inactive fragments. These fragments spontaneously reassociate to form fully active enzyme that, in the assay format, cleaves a substrate, generating a colour change that can be measured spectrophotometrically. In the assay, drug in the sample competes with drug conjugated to the inactive fragment of $\beta$-galactosidase for antibody binding site. If drug is present in the sample, it binds to antibody, leaving the inactive enzyme fragments free to form active enzyme. If drug is not present in the sample, antibody binds to drug conjugated on the inactive fragment, inhibiting the reassociation of inactive $\beta$-galactosidase fragments, and no active enzyme will be formed. The amount of active enzyme formed and resultant absorbance change are proportional to the amount of drug present in the sample.

The Beckman digoxin assay is used to measure the digoxin concentration by a particle enhanced turbidimetric inhibition immunoassay method. Particle-bound drug (PBD) binds to digoxin specific antibody ( $\mathrm{Ab}$ ) resulting in the formation of insoluble aggregates causing increased turbidity. Non-particle-bound digoxin in the patient sample competes with the PBD for the antibody binding sites, inhibiting the formation of insoluble aggregates. The rate and amount of particle aggregation is inversely proportional to the concentration of digoxin in the sample.

According to the manufacturers reagent guide the therapeutic range of phenytoin was $10-20 \mu \mathrm{g} / \mathrm{mL}$, valproic acid $50-100 \mu \mathrm{g} / \mathrm{mL}$, carbamazepine $4-10 \mu \mathrm{g} / \mathrm{mL}$ and digoxin as $0.8-2 \mu \mathrm{g} / \mathrm{L}$.

Statistical determinations were calculated using Microsoft Excel spreadsheets.

\section{RESULTS}

In the 10 months period, a total of 666 TDM tests were performed in the laboratory. 531 of them were to measure one or more AED drugs and 135 were to measure digoxin serum levels. The median age of AEDs TDM patients was $35(95 \% \mathrm{Cl} 32-37)$ years and $52 \%$ of these were female. The median age of patients for digoxin test for TDM was 77 (95\% $\mathrm{Cl} 75-79)$ years and $60 \%$ of these were female. 531 AED tests included 184 carbamazepine, 283 valproic acid and 64 phenytoin measurements. In 11 patients digoxin plus one or more AED levels were measured in the same serum specimen. 8 patients for carbamazepine, 31 patients for valproic acid, 3 patients for phenytoin and 12 patients for digoxin had 2 repeat tests for drug serum levels. 3 patients for carbamazepine, 7 patients for valproic acid, 2 patients for phenytoin and 5 patients for digoxin had 3 repeats for drug serum levels. 4 patients had 4 or more repeats. 
These repeated tests were within the same day or the next day for digoxin, while repeats for AEDs were performed at least 1 month later.

Requests from Neurology Department composed the majority of carbamazepine TDM requests (146 of 184). $70(38 \%)$ of carbamazepine test results were in the therapeutic range, while 101(55\%) were in the subtherapeutic and $13(7 \%)$ were above-therapeutic range. 98 of 101 subtherapeutic carbamazepine test results were requests from Psychiatry Department. 133 of 283 valproic acid TDM requests were from Neurology Department followed by 98 requests from Psychiatry and 23 from Pediatrics Departments. 158 (56\%) of 283 valproic acid test results were in the therapeutic reference range, while $83(29 \%)$ were in the subtherapeutic and $42(15 \%)$ were in abovetherapeutic ranges. 35 of 64 phenitoin TDM requests were made from Neurology Department. $13(20 \%)$ of 64 phenitoin test results were in the therapeutic reference range, while $43(68 \%)$ were in the subtherapeutic and $8(12 \%)$ were in abovetherapeutic ranges. 65 of 135 digoxin tests were from Emergency and Intensive Care Units ( 33 and 32 requests respectively), followed by 27 requests from Nephrology and 13 requests from Cardiology Departments. $53(39 \%)$ of all digoxin tests were in the therapeutic reference range, while $27(20 \%)$ were in the subtherapeutic and $55(41 \%)$ were abovetherapeutic range. Neurology Department made 314 of 531 total AED TDM requests (59\%). 23 of 31 requests from Pediatrics Department were for valproic acid TDM measurements. Percentiles of requests and test results in subtherapeutic, therapeutic and abovetherapeutic ranges are shown in Table 1 and Figures 1 and 2.

\section{DISCUSSION}

Situations that may be appropriate for TDM include dosage adjustment, confirmation of suspected toxicity, identification of non-compliance, management of drug interactions and specific clinical conditions like in the cases of uremic patients, patients with liver disease, pediatric and geriatric patients, critically ill patients and pregnants. ${ }^{2-4}$ High percentage of supra-therapeutic digoxin test results emphasizes the use of TDM for this drug as confirmation of suspected toxicity or overdose. In general, drug overdose is evidenced by patients medical history and clinical findings. However, in some cases of digoxin overdose, the findings may pose a dilemma that the clinician may ask for TDM. Considering the second largest group of requests from Intensive Care Unit, it seems quite clear that clinicians were uncomfortable with critically ill patients using digoxin. The digoxin requests from Nephrology Department were more than the requests from Cardiology Department reflecting the importance of renal functions in digoxin dosage management. Our experience showed that the laboratories should expect multiple digoxin test requests from the same patient in the same day if the test result is out of the desired range. In AED test requests the major clinical concern seems to be dosage management rather than toxicity. In all three parameters of AED TDM measurements high number of results in sub-therapeutic range were of concern. Actually, subtherapeutic test results doubled the total of therapeutic and above-therapeutic test results in phenytoin TDM results and were more than the total of therapeutic and above-therapeutic test results in carbamazepine measurements. Although clinical feedback is lacking, some very low results were suggestive of noncompliance in these patients. In fact, poor patient compliance is one of the major causes of non responsiveness to antiepileptic drug therapy. Compliance is mostly assessed by self-reporting, but in select cases TDM may be very useful in identifying it. ${ }^{7}$ Our valproic acid test results differed from carbamazepine and phenytoin test results with higher percentage of results in therapeutic range.

All AED TDM requests from Physiciatry and Pediatrics Departments were for valproic acid measurements. Phenytoin is not the most common AED in clinical management of epilepsy but is frequently ordered due to its nonlinear pharmacokinetics which makes it very difficult to estimate blood concentration. ${ }^{5}$ Our results were similar to a previuos study from two university hospitals that reported phenytoin test results with highest subtherapeutic percentage. ${ }^{8}$ The distribution of parameters in total AED TDM requests, differs in literature. Irshaid et al. reported phenytoin as the most requested TDM followed by carbamazepine and valproic acid requests'; while in the study by Shakya et al. 241 requests of carbamazepine TDM was followed by 95 for valproic acid and 81 for phenytoin TDM requests. ${ }^{10}$ Figure 1 shows our results, where the number of valproic acid TDM requests were followed by requests for carbamazepine and phenytoin. The contradiction is a clear sign of uncertainity of indications of TDM requesting. Actually, several studies have shown that AED TDM measurements are often requested without an appropriate indication. ${ }^{11,12}$

Currently, a formal request form for TDM is not used in our laboratory. Our automation system, which was the main source of data in this retrospective study, did not give us the information about the sampling time in relation to the dose, the dosage history, the patient's response, AED dose and dosing interval, indication for AED level determination. We are not confident to declare that we were able to measure our performance in this test panel. Affolter et al. reviewed a total of 8057 
Table 1: Sub-therapeutic test results doubled the total of therapeutic and above-therapeutic test results in phenitoin measurements and were more than the total of therapeutic and above-therapeutic test results in carbamazepine measurements, while valproic acid test results were mostly in therapeutic range. Digoxin test results differed from anti-epileptic drug test results with a higher percentage of measurements in abovetherapeutic range

\begin{tabular}{|c|c|c|c|c|}
\hline & Carbamazepine & Valproic acid & Phenitoin & Digoxin \\
\hline $\begin{array}{l}\text { Subtherapeutic } \\
\mathrm{n}(\%) \text { mean } \pm S D\end{array}$ & $101(55 \%) 4,8 \pm 0,4$ & $\begin{array}{c}83(29 \%) \\
26 \pm 18\end{array}$ & $43(68 \%) 3,2 \pm 2,8$ & $27(20 \%) 0,4 \pm 0,2$ \\
\hline $\begin{array}{l}\text { Therapeutic } \\
\mathrm{n}(\%) \text { mean } \pm S D\end{array}$ & $\begin{array}{c}70(38 \%) \\
9,7 \pm 1\end{array}$ & $158(56 \%) 74 \pm 12$ & $13(20 \%) 14,7 \pm 3,9$ & $53(39 \%) 1,01 \pm 0,1$ \\
\hline $\begin{array}{l}\text { Abovetherapeutic } \\
\mathrm{n}(\%) \text { mean } \pm \mathrm{SD}\end{array}$ & $13(7 \%) 14,1 \pm 3,4$ & $42(15 \%) 114 \pm 13$ & $8(12 \%) 40,4 \pm 16,7$ & 55 (41\%) 2,41士1,14 \\
\hline
\end{tabular}

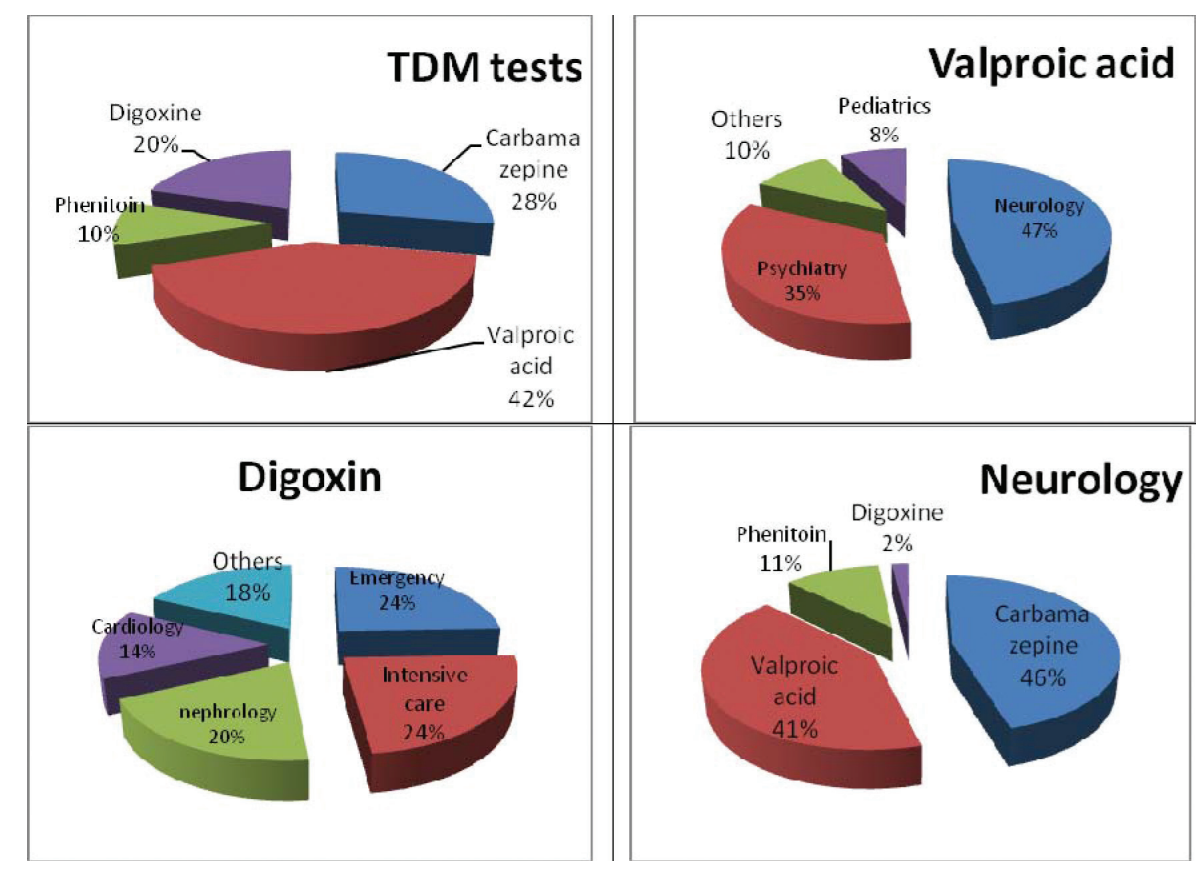

Figure 1: Valproic acid requests composed the majority of total TDM, while Neurology and Psychiatry Clinics made the majority of valproic acid requests. Nearly half of the digoxin TDM requests were from Emergency and Intensive Care units. Valproic acid and carbamazepine requests composed most of AED test requests from Neurology Clinic. Neurology Department made 314 of 531 total AED TDM requests (59\%)

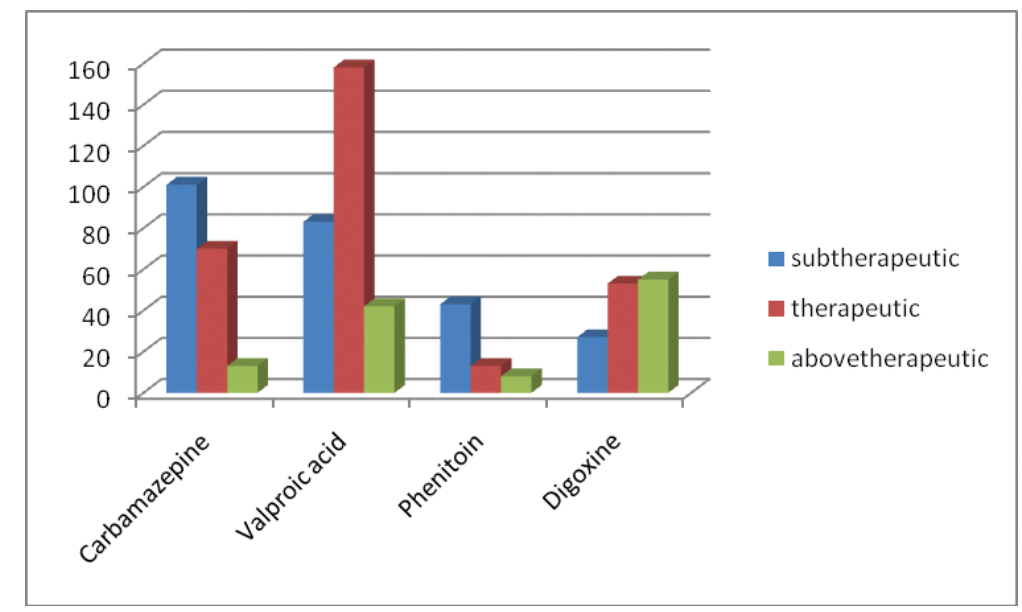

Figure 2: High percentage of abovetherapeutic digoxin test results were in line with the traditional use of TDM in this drug as confirmation of suspected toxicity or overdoseThe test results in subtherapeutic range in all three AED drugs but especially in phenitoin measurements, were the striking feature unlike the high rate of test results in therapeutic range in valproic acid measurements 
TDM results for appropriateness of test requests. ${ }^{11}$ Only $26 \%$ of the tests were with a request form, so the remaining $74 \%$ was excluded in the study. The authors reported an extremely high percentage of appropriateness $(77 \%)$ in their study. Schoenenberger et al. found the percentage of appropriateness as $27 \%$ in their study. ${ }^{13}$ Related to our previous assumption, we think that the excluded majority was a question mark for the reliability of the former study results.

This study of ours may also interest the reader as it is the repetation of a similar one performed two years ago in our hospital by the Clinical Pharmacology Department. ${ }^{14}$ Running some tests including TDM is a matter of debate between departments in our country. Depending on the number of requests in both studies (325 in the former versus 666 in the latter), moving the tests to the Central Laboratory under the control of Clinical Biochemistry, seems to be a good decision in this example. The explosive increase was probably due the higher capability of $7 / 24$ service in our laboratory. We strongly believe in that as the name implies, central laboratory is the most feasible place for instrumental human body fluid analysis. We show extra effort to keep all laboratory analysis in our laboratory. Reporting the test results by a clinical biochemist instead of a clinical pharmacologist did not make a difference since the results were reported without a comment or recommendation. Ideally, TDM results should be evaluated by collaboration of a TDM team, comprised of patients clinicians, other health care stuff like nurses, and laboratory specialist. ${ }^{3}$ Communication among team members is a necessity to achieve the best practices in TDM. The clinician should share the patients information prior to the analysis as well as a feed back on dosage adjustment after the analysis with the TDM team. An appropriate TDM evaluation requires at least the information of sampling time, exact time of the last dose administered and duration of the therapy. The final report should include a written comment on dosage adjustment.

\section{CONCLUSION}

In conclusion, this study will be informative for laboratories that currently do not perform the TDM but planning to do, with respect to drug selection and department negotiations. The current literature on TDM is still contradictory with our results and does not help to compose a future prospect. Contradiction is most likely due to in apppropriate use of TDM by the clinicians.
It is the duty of the laboratory specialist to maintain the appropriateness of TDM. In order to do so, the laboratory must be actively included in all stages of test process starting from acception or rejection of the request. In this study we were very much limited by the absence of critical information like drug history and the patient's clinical condition. We strongly suggest the use of an electronic use of a TDM request form which will unable the test request unless it is properly filled.

\section{CONFLICT OF INTEREST}

The author declares there is no conflict of interest.

\section{ABBREVIATIONS USED}

TDM: Therapeutic drug monitoring

AED: Anti-epileptic drugs

\section{REFERENCES}

1. Duhme DW, Greenblatt DJ, Koch-Weser J. Reduction of digoxin toxicity associated with measurement of serum levels: a report from the Boston Collaborative Drug Surveillance program. Ann Intern Med. 1974;80(4):516-9.

2. Kang JS, Lee MH. Overview of Therapeutic Drug Monitoring. Korean J Intern Med. 2009;24(1):1-10.

3. Ratanajamit C, Kaewpibal P, Setthawacharavanich S, Faroongsarng D. Effect of pharmacist participation in the health care team on therapeutic drug monitoring utilization for antiepileptic drugs. J Med Assoc Thai. 2009;92(11):1500-7.

4. Ghiculescu RA. Therapeutic drug monitoring: which drugs, why, when and how to do it. Aust Prescr. 2008;31(2):42-4.

5. Jannuzzi G, Cian P, Fattore C, Gatti G, Bartoli A, Monaco F, et al. A multicenter randomized controlled trial on the clinical impact of therapeutic drug monitoring in patients with newly diagnosed epilepsy. The Italian TDM Study Group in Epilepsy. Epilepsia. 2000;41(2):222-30.

6. Tomson T, Dahl ML, Kimland E. Therapeutic monitoring of antiepileptic drugs for epilepsy. Cochrane Database Syst Rev. 2007;24(1):CD002216.

7. Chandra RS, Dalvi SS, Karnad PD, Kshirsagar NA, Shah PU. Compliance monitoring in epileptic patients. J Assoc Physicians India. 1993;41(7):431-2.

8. Koristkova B, Bergman U, Grundmann M, Brozmanova $H$, Sjöqvist $F$. Therapeutic monitoring of antiepileptic drugs: a comparison between a Czech and a Swedish University Hospital. Ther Drug Monit. 2006;28(5):594-8.

9. Irshaid YM, Hamdi AA, Al Homrany M. Evaluation of therapeutic drug monitoring of antiepileptic drugs. Int J Clin Pharmacol Ther. 2003;41(3):126-31.

10. Shakya G, Malla S, Shakya KN, Shrestha R. Therapeutic drug monitoring of antiepileptic drugs. JNMA J Nepal Med Assoc. 2008;47(171):94-7.

11. Affolter N, Krahenbühl S, Schlinger RG. Appropriateness of serum level determinations of antiepileptic drugs. Swiss Med Wkly. 2003;133(43-44):5917.

12. Sharma S, Joshi S, Mukherji S, Bala K, Tripathi CB. Therapeutic drug monitoring: appropriateness and clinical utility in neuropsychiatry practice. Am J Ther. 2009;16(1):11-6.

13. Schoenenberger RA, Tanasijevic MJ, Jha A, Bates DW. Appropriateness of antiepileptic drug level monitoring. JAMA. 1995;274(20):1622-6.

14. Dalaklıglu S. Evaluating Appropriateness of Digoxin, Carbamazepine, Valproic Acid, and Phenytoin Usage by Therapeutic Drug Monitoring. Clin Lab. 2013;59(3-4):325-31. 